\title{
Clinical Diagnosis of Placenta Accreta and Clinicopathological Outcomes
}

\author{
Joshua I. Rosenbloom, MD, MPH ${ }^{1}$ Jonathan S. Hirshberg, $\mathrm{MD}^{1}$ Molly J. Stout, $\mathrm{MD}, \mathrm{MSCl}^{1}$ \\ Alison G. Cahill, MD, MSCl ${ }^{1}$ George A. Macones, MD, MSCE${ }^{1}$ Methodius G. Tuuli, MD, MPH² \\ ${ }^{1}$ Department of Obstetrics and Gynecology, Washington University \\ Address for correspondence Joshua I. Rosenbloom, MD, MPH, \\ in St. Louis School of Medicine, St. Louis, Missouri \\ Department of Obstetrics and Gynecology, Washington University in \\ 2 Department of Obstetrics and Gynecology, Indiana University \\ School of Medicine, Indianapolis, Indiana \\ St. Louis School of Medicine, 660 South Euclid Avenue Campus Box \\ 8064, Saint Louis, MO 63110 \\ (e-mail: rosenbloomj@wudosis.wustl.edu).
}

Am J Perinatol 2019;36:124-129.

\begin{abstract}
Objective To investigate the association between the intraoperative diagnosis of placenta accreta at the time of cesarean hysterectomy and pathological diagnosis. Study Design This is a retrospective cohort study of all patients undergoing cesarean hysterectomy for suspected placenta accreta from 2000 to 2016 at Barnes-Jewish Hospital. The primary outcome was the presence of invasive placentation on the pathology report. We estimated predictive characteristics of clinical diagnosis of placenta accreta using pathological diagnosis as the correct diagnosis.

Results There were 50 cesarean hysterectomies performed for suspected abnormal placentation from 2000 to 2016. Of these, 34 (68\%) had a diagnosis of accreta preoperatively and $16(32 \%)$ were diagnosed intraoperatively at the time of cesarean delivery. Two patients had no pathological evidence of invasion, corresponding to a false-positive rate of $4 \%$ (95\% confidence interval [Cl]: $0.5 \%, 13.8 \%)$ and a positive predictive value of $96 \%(95 \% \mathrm{Cl}: 86.3 \%, 99.5 \%)$. There were no differences in

Keywords

- placenta accreta

- cesarean hysterectomy

- clinicopathological outcomes

- clinical diagnosis complications among patients diagnosed intraoperatively compared with those diagnosed preoperatively.

Conclusion Most patients undergoing cesarean hysterectomy for placenta accreta do have this diagnosis confirmed on pathology. However, since the diagnosis of placenta accreta was made intraoperatively in nearly a third of cesarean hysterectomies, intraoperative vigilance is required as the need for cesarean hysterectomy may not be anticipated preoperatively.
\end{abstract}

As the rates of cesarean delivery increase, there has been a proportional increase in the rates of placenta accreta. ${ }^{1}$ Clinically, placenta accreta is defined by placental invasion of the uterine wall or an inability to separate the placenta from the uterine wall at the time of delivery. ${ }^{2}$ Pathologically, placenta accreta, increta, and percreta are diagnosed when the placenta extends through the decidua basalis and into the myometrium or uterine serosa, respectively. ${ }^{3}$ Patients with placenta accreta are at a high risk of maternal morbidity or mortality at the time of delivery and usually require cesarean hysterectomy. ${ }^{3}$

received

January 10, 2018

accepted after revision

August 7, 2018

published online

September 7, 2018
However, even despite advances in imaging and greater understanding of risk factors, rates of preoperative diagnosis of placenta accreta remain low, 30 to $80 \%$, depending on the practice setting and country, with a recent rate of $50 \%$ reported from a large United States database. ${ }^{4}$ This means that obstetrician-gynecologists are often making split-second decisions regarding the need for the morbid and sterilizing procedure of hysterectomy. ${ }^{5-7}$ Further research is needed to understand the accuracy and consequences of these decisions. ${ }^{5,8-14}$

Copyright @ 2019 by Thieme Medical Publishers, Inc., 333 Seventh Avenue, New York, NY 10001, USA. Tel: +1(212) 584-4662.
DOI https://doi.org/ 10.1055/s-0038-1670635. ISSN 0735-1631. 
Therefore, the objectives of this study were to (1) investigate the relative frequency of preoperative versus intraoperative diagnosis of placenta accreta, (2) investigate the association between the diagnosis of placenta accreta at the time of cesarean hysterectomy and pathological diagnosis, and (3) test the hypothesis that compared with intraoperative diagnosis, preoperative diagnosis of placenta accreta is associated with a lower risk of intraoperative complications.

\section{Materials and Methods}

This is a retrospective cohort study of patients undergoing cesarean hysterectomy for suspected placenta accreta. All patients undergoing cesarean hysterectomy from 2000 to 2016 at Washington University School of Medicine in St. Louis for suspected placenta accreta were included. Placenta accreta was defined as placental invasion to (accreta), into (increta), or through (percreta) the myometrial wall. ${ }^{2}$ Patients were identified by searching the comprehensive billing database for relevant ICD-9 (International Statistical Classification of Diseases and Related Health Problems) and CPT (Current Procedural Terminology) codes capturing hysterectomy at the time of cesarean and placenta accreta. Patients were excluded if they underwent cesarean hysterectomy for other indications (e.g., uterine rupture, atony) or if placenta pathology reports were unavailable. The electronic medical records of all eligible patients were reviewed. We abstracted patient and pregnancy characteristics including preoperative diagnoses, imaging studies when available, intraoperative findings, and placental pathology results. The operative report (specifically the listed preoperative diagnosis, postoperative diagnosis, and operative findings) was used to determine the preoperative diagnosis as well as the intraoperative findings. In cases of ambiguity, the report was reviewed by multiple authors to achieve consensus.

We defined "clinical diagnosis" as any nonpathological diagnosis (made either preoperatively or intraoperatively), "preoperative diagnosis" as having placenta accreta listed as a preoperative diagnosis on the operative report or included in the indications for procedure, and "intraoperative diagnosis" as a clinical diagnosis first made at the time of cesarean section.

Patient and pregnancy information included maternal age, gestational age at delivery, race, height and weight at the time of delivery, obstetric history, number of prior cesarean deliveries, multiple pregnancies, tobacco use, and presence of placenta previa. Gestational age was based on the best obstetrical estimate as recorded in the medical record. All types of placenta previa (marginal, complete, etc.) were categorized as placenta previa. Imaging data included ultrasound and, in some cases, magnetic resonance imaging (MRI) reports. Pathological diagnoses were determined from the pathology report. In cases of multiple levels of invasion, the most severe was used (e.g., "placenta accreta with focal increta" was coded as "increta"). Surgical findings and complications were based on the operative report. The type of surgery was categorized as either cesarean total hysterectomy or cesarean supracervical hysterectomy. Intraoperative complications were categorized as cystotomy, ureteral injury, or other. Intentional cystotomies were recorded as "cystotomy." Admission to the intensive care unit was noted. Estimated blood loss was obtained from the operative report. Intraoperative blood transfusion of packed red blood cells was recorded in units based on the operative report and anesthesia record.

The primary outcome was the diagnosis of invasive placentation in the pathology report. Secondary outcomes were estimated blood loss and intraoperative complications. Test characteristics including positive predictive value and falsepositive rate with $95 \%$ confidence intervals (CIs) of clinical diagnosis were estimated with the pathology diagnosis of placenta accreta as the gold standard. Patients were categorized into two groups based on the timing of diagnosis of placenta accreta: preoperative or intraoperative, as discussed previously. Baseline characteristics and outcome measures were compared with the Mann-Whitney $U$-test or Fisher's exact test as appropriate.

Study data were collected and managed using REDCap electronic data capture tools hosted at the Washington University School of Medicine in St. Louis. ${ }^{15}$ The study was approved by the Washington University in St. Louis Human Research Protection Office. Given that we included all women who met inclusion criteria and the sample size was fixed, we did not perform an a priori sample size calculation. Analyses were performed in SAS software (version 9.2, SAS Institute Inc., Cary, NC). A $p$-value of $<0.05$ was considered statistically significant. We did not adjust for multiple comparisons.

\section{Results}

From 2000 to 2016, 80 cesarean hysterectomies were performed: 52 of which were for suspected placenta accreta (2 had a missing pathology report, leaving a final sample size of 50)(-Fig. 1).The majority of these were in the years from 2013 to $2016(n=28)$, with the remainder distributed relatively evenly over the preceding years. Of the 50 patients, 34 (68\%) had a preoperative diagnosis of placenta accreta, whereas the diagnosis was first made intraoperatively in the remaining 16 (32\%) at the time of cesarean delivery. The majority of patients $(n=38 ; 76 \%)$ had a preoperative diagnosis of placenta previa and at least one prior cesarean delivery.

Baseline characteristics were similar among patients diagnosed preoperatively and intraoperatively, with the notable exception that placenta previa and prior cesarean were more common among patients diagnosed preoperatively: placenta previa (97 vs. $44 \% ; p<0.0001$ ) and mean number of prior cesareans ( 3 vs. $2 ; p=0.02$ ) ( - Table 1 ).

In all, 48 (96\%) patients had confirmed placenta accreta in the pathology report, whereas 2 (4\%) patients had no evidence of invasion. This corresponds to an overall positive predictive value of a clinical diagnosis of $96 \%(95 \% \mathrm{CI}: 86.3 \%, 99.5 \%)$, and a false-positive rate of $4 \%$ (95\% CI: $0.5 \%, 13.8 \%$ ) (-Table 2).

Of the 16 patients with initial intraoperative diagnosis, 9 (56\%) had an initial attempt to remove the placenta. The remainder did not have such an attempt due to visibly abnormal placentation at the time of the delivery. All patients who were first diagnosed intraoperatively $(n=16)$ had pathological confirmation of placenta accreta. 


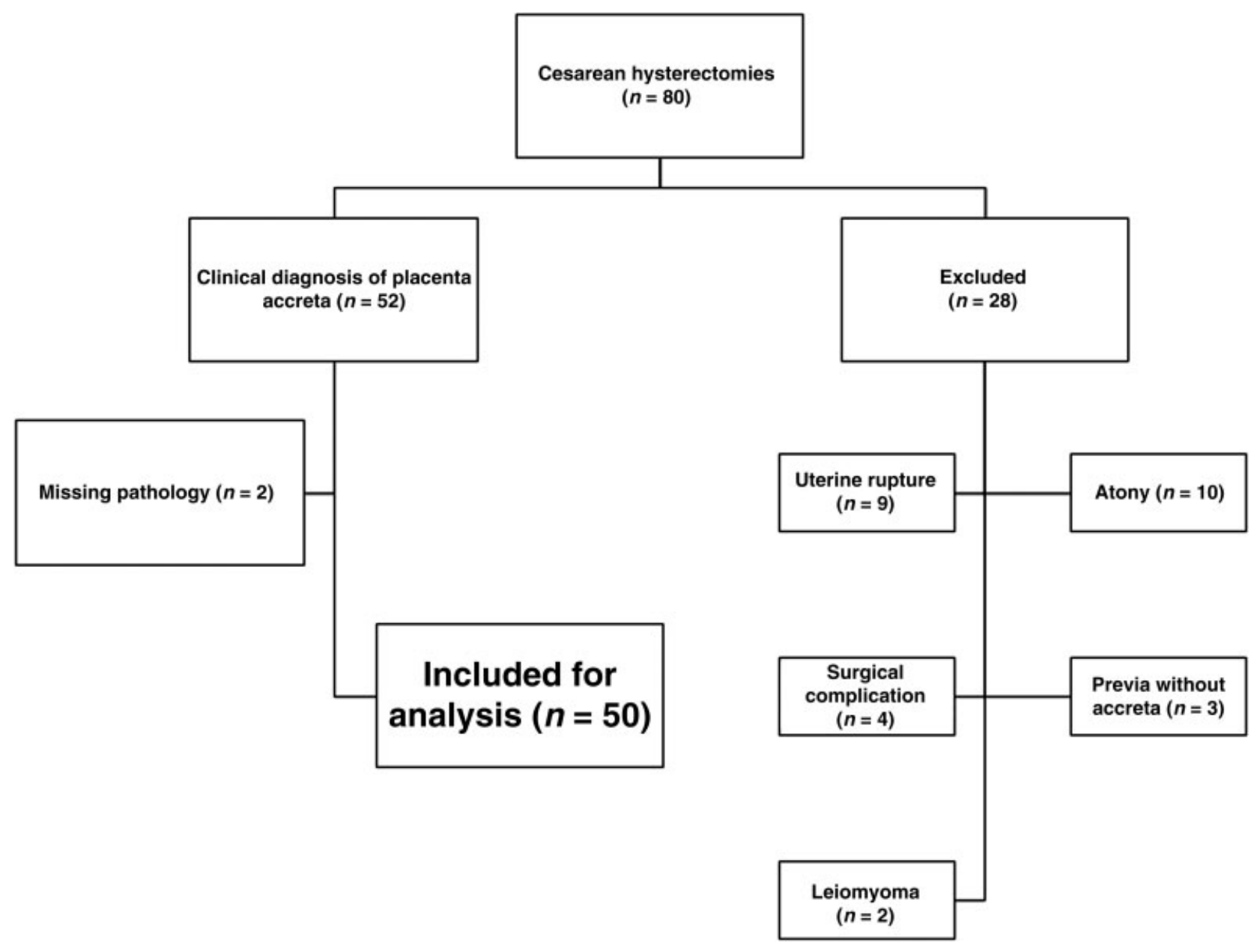

Fig. 1 Study flow diagram.

Table 1 Preoperative characteristics $(n=50)$

\begin{tabular}{|c|c|c|c|c|}
\hline Characteristic & $\begin{array}{l}\text { Entire cohort } \\
(n=50)\end{array}$ & $\begin{array}{l}\text { Invasion diagnosed } \\
\text { preoperatively } \\
(n=34)\end{array}$ & $\begin{array}{l}\text { Invasion first diagnosed } \\
\text { intraoperatively } \\
(n=16)\end{array}$ & $p$-Value ${ }^{a}$ \\
\hline Maternal age, years & $31.4(5.9)$ & $31.7(5.3)$ & $30.8(7.2)$ & 0.44 \\
\hline Gestational age at delivery, weeks & $33(3.1)$ & $33.4(3.2)$ & $32.3(3.8)$ & 0.24 \\
\hline Race & & & & 0.72 \\
\hline Black & $17(34)$ & $10(29)$ & $7(44)$ & \\
\hline Caucasian & $29(58)$ & $21(62)$ & $8(50)$ & \\
\hline Other & $4(8)$ & $3(9)$ & $1(6)$ & \\
\hline Body mass index $\left(\mathrm{kg} / \mathrm{m}^{2}\right)$ & $31.5(7.3)$ & $31.3(8.2)$ & $31.9(5.1)$ & 0.44 \\
\hline Gravidity & $5(2)$ & $5(2)$ & $4(1)$ & 0.04 \\
\hline Parity & $3(2)$ & $3(2)$ & $2(1)$ & 0.16 \\
\hline Prior cesarean delivery & $46(94)$ & $32(94)$ & $14(88)$ & 0.58 \\
\hline Number of prior cesareans & $2(1)$ & $3(2)$ & $2(1)$ & 0.02 \\
\hline Multiple pregnancy & $4(8)$ & $1(3)$ & $3(18)$ & 0.09 \\
\hline Tobacco use & $8(16)$ & $6(18)$ & $2(13)$ & 1 \\
\hline Placenta previa & $40(80)$ & $33(97)$ & $7(44)$ & $<0.0001$ \\
\hline
\end{tabular}

${ }^{a}$ Values using Mann-Whitney U-test or Fisher's exact test.

Note: Data are presented as $n$ (\%) or mean (standard deviation). 
Table 2 Pathological outcomes $(n=50)$

\begin{tabular}{|l|l|}
\hline Outcome & $\boldsymbol{n}(\%)$ \\
\hline No invasion & $2(4)$ \\
\hline Accreta & $19(38)$ \\
\hline Increta & $18(36)$ \\
\hline Percreta & $11(22)$ \\
\hline
\end{tabular}

- Table 3 shows the relationship between intraoperative diagnosis of the degree of abnormality and the pathological diagnosis. Of the two false-positive clinical diagnoses of placental invasion, one was thought to be a placenta accreta while the other was a suspected placenta increta. Both patients had a preoperative ultrasound diagnosis of placenta accreta.

Most patients (84\%) had total cesarean hysterectomy, and the remainder (16\%) had supracervical hysterectomy. There was no difference in total hysterectomy rates in patients with preoperative and postoperative diagnoses of placenta accreta ( 88 vs. $75 \% ; p=0.25$ ). The median blood loss was $2,500 \mathrm{~mL}$ for the entire cohort, with mean hospital length of stay of 4.5 days, with no differences between the groups diagnosed preoperatively versus intraoperatively. Nearly one in five women were admitted to the intensive care unit, with no differences in those diagnosed preoperatively and intraoperatively ( 21 vs. $13 \% ; p=0.70$ ) ( - Table 4). There were no maternal deaths. Intraoperative surgical injury was common, with nearly one in three patients sustaining a cystostomy, ureteral injury, or other type of injury ( $n=16 ; 32 \%)$.

\section{Discussion}

We found that nearly one-third of diagnoses of placenta accreta were only made at the time of delivery. Additionally, although overall clinical diagnosis (preoperative and intraoperative) was largely accurate, there was a $4 \%$ falsepositive rate where the clinical diagnosis of placenta accreta was not confirmed on pathological examination. There were no significant differences in outcomes between patients diagnosed preoperatively and those diagnosed intraoperatively.

These findings indicate that when faced with a "surprise" potential placenta accreta, clinical diagnosis is highly accurate. The majority of the 16 patients with initial intraoperative diagnosis had an initial attempt to remove the placenta, suggesting that the diagnosis was indeed unexpected. Notably, all of these patients had pathological confirmation of

Table 3 Relationship between intraoperative and pathological diagnoses $(n=50)$

\begin{tabular}{|c|c|c|c|c|c|}
\hline & & \multicolumn{4}{|c|}{ Pathological diagnosis } \\
\hline \multirow[t]{4}{*}{ Intraoperative diagnosis } & & No invasion & Accreta & Increta & Percreta \\
\hline & Accreta & $1(2)$ & $14(28)$ & $13(26)$ & $4(8)$ \\
\hline & Increta & $1(2)$ & $1(2)$ & $3(6)$ & $2(4)$ \\
\hline & Percreta & $0(0)$ & $4(8)$ & $2(4)$ & $5(10)$ \\
\hline
\end{tabular}

Table 4 Surgical outcomes $(n=50)$

\begin{tabular}{|c|c|c|c|c|}
\hline Outcome & Entire cohort & $\begin{array}{l}\text { Invasion diagnosed } \\
\text { preoperatively } \\
(n=34)\end{array}$ & $\begin{array}{l}\text { Invasion diagnosed } \\
\text { intraoperatively } \\
(n=16)\end{array}$ & $p$-Value \\
\hline Surgical procedure & & & & 0.25 \\
\hline Cesarean total hysterectomy & $42(84)$ & $30(88)$ & $12(75)$ & \\
\hline $\begin{array}{l}\text { Cesarean supracervical } \\
\text { hysterectomy }\end{array}$ & $8(16)$ & $4(12)$ & $4(25)$ & \\
\hline Intraoperative complication & $16(32)$ & $14(41)$ & $2(12)$ & 0.26 \\
\hline Cystotomy & $13(26)$ & $11(32)$ & $2(13)$ & \\
\hline Ureteral injury & $1(2)$ & $1(3)$ & $0(0)$ & \\
\hline Other & $2(4)$ & $2(6)$ & $0(0)$ & \\
\hline Intensive care unit admission & $9(18)$ & $7(21)$ & $2(13)$ & 0.70 \\
\hline Estimated blood loss (mL) & $2,400(1,500-3,100)$ & $2,600(1,500-3,500)$ & $2,000(1,250-2,750)$ & 0.13 \\
\hline $\begin{array}{l}\text { Packed red blood cells transfused } \\
\text { intraoperatively (units) }\end{array}$ & $2.5(1-4)$ & $3(1-5)$ & $2(0.5-4)$ & 0.37 \\
\hline $\begin{array}{l}\text { Length of postoperative admission, } \\
\text { days, mean (standard deviation) }\end{array}$ & $4.5(1.2)$ & $4.7(1.4)$ & $4.1(0.6)$ & 0.22 \\
\hline
\end{tabular}

Note: Data are presented as $n$ (\%) or median (interquartile range). p-Values derived using Mann-Whitney U-test or Fisher's exact test comparing preoperative and intraoperative diagnosis groups. 
placenta accreta, further suggesting that the abandonment of the placenta delivery attempt and the decision to perform hysterectomy was justified.

The proportion of intraoperative versus preoperative diagnosis of placenta accreta in our study were similar to those in other studies, which have ranged from a preoperative diagnosis rate of 30 to $80 \% .^{5,6,10,14,16}$ In a retrospective case series of 99 pathologically confirmed placenta accreta cases, $63 \%$ were diagnosed antepartum and the remaining $37 \%$ diagnosed at delivery. ${ }^{14}$ On the other hand, in a recent study of 205 cases from the Nordic countries, $70 \%$ were undiagnosed preoperatively. ${ }^{5}$ However, that study considered only clinical diagnosis and not pathological diagnosis. In our study, patients with risk factors were more likely to be diagnosed preoperatively, perhaps reflecting provider bias in more vigilantly searching for placenta accreta in patients with risk factors.

Further study is needed to improve rates of preoperative diagnosis. ${ }^{17}$ The diagnostic accuracy of ultrasound for abnormal placentation is variable, with a recent report citing a false-negative rate as high as $16 \%{ }^{10}$ However, a meta-analysis noted that in experienced units, ultrasound is highly sensitive and specific for the diagnosis of placenta accreta with prenatal detection rates of $>90 \%{ }^{11}$ This variability in the accuracy of ultrasound diagnosis of abnormal placentation is likely because of the operator-dependent nature of ultrasound examinations.

It is recognized that patients with placenta accreta have better outcomes when treated in a specialty center with multidisciplinary care. $^{7-9}$ However, it is unclear to what degree the patients managed by regular care in these studies had a suspected diagnosis prior to surgery. ${ }^{8}$ Notably, a recent study from across the United States actually found worse outcomes in patients with a preoperative diagnosis compared with an initial intraoperative diagnosis, perhaps due to more severe disease. ${ }^{4}$ The lack of significant differences in outcomes between patients with preoperative diagnosis and those with intraoperative diagnosis in our study may be attributable to the fact that patients were treated in a regional perinatal health care center with continuous access to advanced pelvic surgeons as well as robust intensive care and blood bank services. ${ }^{18}$ Thus, outcomes may not be the same as in a community setting where an unplanned cesarean hysterectomy confers significantly more morbidity. ${ }^{9}$

There were several strengths of our study. The study was conducted in an institution with common approaches to the diagnosis and management of placenta accreta. In addition, detailed data were abstracted, allowing for comprehensive analysis. However, there are limitations to be considered when interpreting our results. This is a retrospective cohort study based on chart review of patients identified through billing codes. While we used exhaustive codes to in an attempt to identify all patients meeting the inclusion criteria, there is a possibility that some patients were missed. Moreover, accuracy of the distinction between preoperative and intraoperative diagnosis of placenta was based on the listed diagnoses and descriptions in the operative reports, which may reflect provider bias. Although our sample size is comparable to prior studies, it is relatively small and may have impacted our ability to find statistically significant differences. ${ }^{1,14,16}$ Finally, we did not have information about preoperative diagnoses of placenta accreta that were not clinically corroborated intraoperatively and therefore did not lead to cesarean hysterectomy. This made it impossible to estimate the test characteristics of preoperative imaging and diagnosis.

In conclusion, in our cohort, the diagnosis of placenta accreta was first made intraoperatively in nearly a third of cesarean hysterectomies. While most patients undergoing cesarean hysterectomy for clinically diagnosed placenta accreta had the diagnosis confirmed on pathological examination, there was a $4 \%$ false-positive rate. When faced with a clinical situation concerning for placenta accreta, clinicians can be reassured that clinical diagnosis is highly accurate. These findings reinforce the need for vigilance intraoperatively as the need for cesarean hysterectomy may not be anticipated preoperatively. As summarized in a recent editorial by Nageotte, clinicians must remain alert and ready for the potential for an undiagnosed placenta accreta at all times. $^{19}$

\section{Funding}

This work was supported by the National Institutes of Health (grant numbers UL1 TR000448 and P30 CA09 1842). Dr. Tuuli is supported by U01HD077384-03 and 1R01HD086007-01 grants. The contents of this publication are solely the responsibility of the authors and do not necessarily represent the official view of the National Institutes of Health. The funder had no role in the study design, collection, analysis, or interpretation of data, in the writing of the report, or in the decision to submit the article for publication.

\section{Conflict of Interest}

None.

\section{References}

1 Miller DA, Chollet JA, Goodwin TM. Clinical risk factors for placenta previa-placenta accreta. Am J Obstet Gynecol 1997; 177(01):210-214

2 ACOG Committee on Obstetric Practice. ACOG Committee opinion. Number 266, January 2002: placenta accreta. Obstet Gynecol 2002;99(01):169-170

3 Belfort MA; Publications Committee, Society for Maternal-Fetal Medicine. Placenta accreta. Am J Obstet Gynecol 2010;203(05): 430-439

4 Bailit JL, Grobman WA, Rice MM, et al; Eunice Kennedy Shriver National Institute of Child Health and Human Development (NICHD) Maternal-Fetal Medicine Units (MFMU) Network. Morbidly adherent placenta treatments and outcomes. Obstet Gynecol 2015;125(03):683-689

5 Thurn L, Lindqvist PG, Jakobsson M, et al. Abnormally invasive placenta-prevalence, risk factors and antenatal suspicion: results from a large population-based pregnancy cohort study in the Nordic countries. BJOG 2016;123(08):1348-1355

6 Fitzpatrick KE, Sellers S, Spark P, Kurinczuk JJ, Brocklehurst P, Knight $\mathrm{M}$. The management and outcomes of placenta accreta, increta, and percreta in the UK: a population-based descriptive study. BJOG 2014;121(01):62-70 
7 Shamshirsaz AA, Fox KA, Salmanian B, et al. Maternal morbidity in patients with morbidly adherent placenta treated with and without a standardized multidisciplinary approach. Am J Obstet Gynecol 2015;212(02):218.e1-218.e9

8 Eller AG, Bennett MA, Sharshiner M, et al. Maternal morbidity in cases of placenta accreta managed by a multidisciplinary care team compared with standard obstetric care. Obstet Gynecol 2011;117(2 Pt 1):331-337

9 Silver RM, Fox KA, Barton JR, et al. Center of excellence for placenta accreta. Am J Obstet Gynecol 2015;212(05):561-568

10 Bowman ZS, Eller AG, Kennedy AM, et al. Accuracy of ultrasound for the prediction of placenta accreta. Am J Obstet Gynecol 2014; 211(02):177.e1-177.e7

11 Jauniaux E, Bhide A. Prenatal ultrasound diagnosis and outcome of placenta previa accreta after cesarean delivery: a systematic review and meta-analysis. Am J Obstet Gynecol 2017;217(01): 27-36

12 Palacios Jaraquemada JM, Bruno $\mathrm{CH}$. Magnetic resonance imaging in 300 cases of placenta accreta: surgical correlation of new findings. Acta Obstet Gynecol Scand 2005;84(08):716-724
13 Warshak CR, Eskander R, Hull AD, et al. Accuracy of ultrasonography and magnetic resonance imaging in the diagnosis of placenta accreta. Obstet Gynecol 2006;108(3 Pt 1):573-581

14 Warshak CR, Ramos GA, Eskander R, et al. Effect of predelivery diagnosis in 99 consecutive cases of placenta accreta. Obstet Gynecol 2010;115(01):65-69

15 Harris PA, Taylor R, Thielke R, Payne J, Gonzalez N, Conde JG. Research electronic data capture (REDCap)-a metadata-driven methodology and workflow process for providing translational research informatics support. J Biomed Inform 2009;42(02):377-381

16 Tikkanen M, Paavonen J, Loukovaara M, Stefanovic V. Antenatal diagnosis of placenta accreta leads to reduced blood loss. Acta Obstet Gynecol Scand 2011;90(10):1140-1146

17 Jauniaux E, Silver RM. Moving from intra partum to prenatal diagnosis of placenta accreta: a quarter of a century in the making but still a long road to go. BJOG 2017;124(01):96

18 Obstetric Care Consensus No. Obstetric Care Consensus No. 2: levels of maternal care. Obstet Gynecol 2015;125(02):502-515

19 Nageotte MP. Always be vigilant for placenta accreta. Am J Obstet Gynecol 2014;211(02):87-88 